\title{
Desbaric air embolism during diving: an unusual complication of Osler-Weber-Rendu disease
}

\section{Y-L Hsu, H-C Wang, P-C Yang}

Br J Sports Med 2004;38:e6 (http://www.bjsportmed.com/cgi/content/full/38/4/e6). doi: 10.1136/bjsm.2003.004432

Cerebral manifestations of Osler-Weber-Rendu disease (OWRD, hereditary haemorrhagic telangiectasia) including telangiectases, venous malformations, and arteriovenous malformations, are usually under-recognised. The highest complication rate is observed in high flow cerebral arteriovenous malformations, which may present with headache, epilepsy, ischaemia, or haemorrhage. Cerebral air embolism during self-contained underwater breathing apparatus (scuba) diving as the first manifestation of pulmonary arteriovenous malformation (PAVM) in OWRD patients has never been reported before. Here we report a 31 year old male who presented desbaric air embolism as the first manifestation of PAVM. As far as we know, this is the first such case published in English medical literature.

$\mathrm{P}$ ulmonary arteriovenous malformations (PAVM) are caused by abnormal communications between pulmonary arteries and pulmonary veins. Although these lesions are quite uncommon, they are an important part of the differential diagnosis of common pulmonary problems such as hypoxemia and pulmonary nodules. Approximately $70 \%$ of cases of PAVM are associated with Osler-Weber-Rendu disease (OWRD, hereditary haemorrhagic telangiectasia). Conversely, approximately $15-35 \%$ of patients with OWRD have PAVM. According to a previous literature review, the clinical manifestations of PAVM are: epistaxis, dyspnoea, telangiectases, bruit, clubbing, cyanosis, haemoptysis, and embolic complications. However, $9-28 \%$ of the patients were asymptomatic when the diagnosis was made. Osler-WeberRendu disease with pulmonary arteriovenous malformation, a cause of extracardiac right-to-left shunt, has never been reported as a risk factor for desbaric air embolism during diving. We report the case of a young man with previously undiagnosed Osler-Weber-Rendu disease who presented with sudden loss of consciousness during diving due to desbaric air embolism.

\section{CASE REPORT}

This 31 year old male patient denied any major systemic disease history. He enjoyed an uneventful life with no exercise intolerance, exertional dyspnoea, or growth retardation during childhood. He joined a diving club and performed self-contained underwater breathing apparatus (scuba) diving at a depth of 3 feet three times without accident 2 years ago. He participated in a hotel hosted diving course on a tropical vacation and experienced sudden loss of consciousness while he was ascending from scuba diving at a depth of 5 feet on August 15th 2002, during the first dive on the first day of his vacation. He was sent to our hospital immediately, and a comatose condition (Glasgow coma scale E1M2V1) accompanied by left side weakness was found during physical examination. His blood pressure was 120/
$90 \mathrm{~mm} \mathrm{Hg}$, his heart rate $92 \mathrm{bpm}$, and his respiratory rate 16 bpm. Oxygen saturation $\left(\mathrm{SaO}_{2}\right)$ was $98 \%$ when breathing room air. There was neither murmur nor bruit over the thorax, but multiple small $(0.2-0.5 \mathrm{~cm})$ telangiectases were found on the lower lip and both palms. Clubbing of the fingers and toes was also found, but no cyanosis was noted. Complete blood count showed a normal haemogram with haemoglobin $14.8 \mathrm{gm} / \mathrm{dl}$, haematocrit $42.8 \%$, platelet $242 \mathrm{k} / \mu \mathrm{l}$, and WBC $4870 / \mu \mathrm{l}$ with normal differentiation. Arterial blood gas showed $\mathrm{pH}$ 7.35, $\mathrm{PCO}_{2}$ : $34.1 \mathrm{~mm} \mathrm{Hg}$, and $\mathrm{PO}_{2}: 96.5 \mathrm{~mm} \mathrm{Hg}$ when breathing room air $\left(\mathrm{DA}-\mathrm{aO}_{2}\right.$ : $10.6 \mathrm{~mm} \mathrm{Hg}$ ). Bilateral multiple nodules over the right upper and left lower lungs were found on chest radiography (fig 1). Brain computed tomography (CT) revealed diffuse swelling of the bilateral hemispheres (fig 2) and desbaric cerebral air embolism was diagnosed. He was treated with endotracheal intubation, osmotic agents, and hyperbaric oxygenation therapy. His consciousness improved gradually and endotracheal extubation was performed 4 days later.

On admission, contrast enhanced CT of the chest also revealed multiple subpleural arteriovenous communications (fig 3). Transthoracic echocardiography was performed which revealed good contractility of bilateral ventricles without intracardiac right-to-left shunt. Chest sonography revealed a $0.6 \times 0.6 \mathrm{~cm}$ subpleural nodule over the left lower lobe, with homogenous, hypoechoic internal echogenicity. A mosaic pattern with mixed high and low velocity was found by colour Doppler ultrasonography (ATL HDI-3000, Advanced Technology Laboratories, Bothell, WA, USA). Spectral waveform (time-velocity Doppler spectra of flow signal) analysis revealed the following Doppler indices: (1) peak systolic velocity: $38.6 \mathrm{~cm} / \mathrm{s} ;(2)$ end diastolic velocity: $17.8 \mathrm{~cm} / \mathrm{s}$; (3) pulsatility index (peak systolic velocity-end diastolic velocity)/mean velocity: 1.82; and (4) resistive (Pourcleot) index (peak systolic velocity-end diastolic velocity)/peak systolic velocity: 0.53 . These findings were compatible with the diagnosis of PAVM by colour Doppler ultrasonography. An angiodysplasia lesion at the upper body, posterior wall of the stomach was also found by upper gastrointestinal panendoscopy. A technetium-99m ( ${ }^{99 \mathrm{~m}} \mathrm{Tc}$ ) methylene diphosphonate whole body bone scan revealed increased uptake in the right humerus and suspected osteonecrosis of the right humeral head, neck, and upper shaft, most possibly due to an air embolism. A ${ }^{99 \mathrm{~m}}$ Tc pertechneate labelled macroaggregated albumin scan revealed intrapulmonary uptake of the brain and both kidneys, which was compatible with right-to-left shunting. Cerebral angiography and contrast enhanced magnetic resonance angiography of the brain revealed no evidence of cerebral arteriovenous malformation or aneurysm. Tracing the patient's history, he had suffered from frequent nose bleeds since childhood, as had his grandfather

Abbreviations: OWRD, Osler-Weber-Rendu disease; PAVM, pulmonary arteriovenous malformation; scuba, self-contained underwater breathing apparatus 


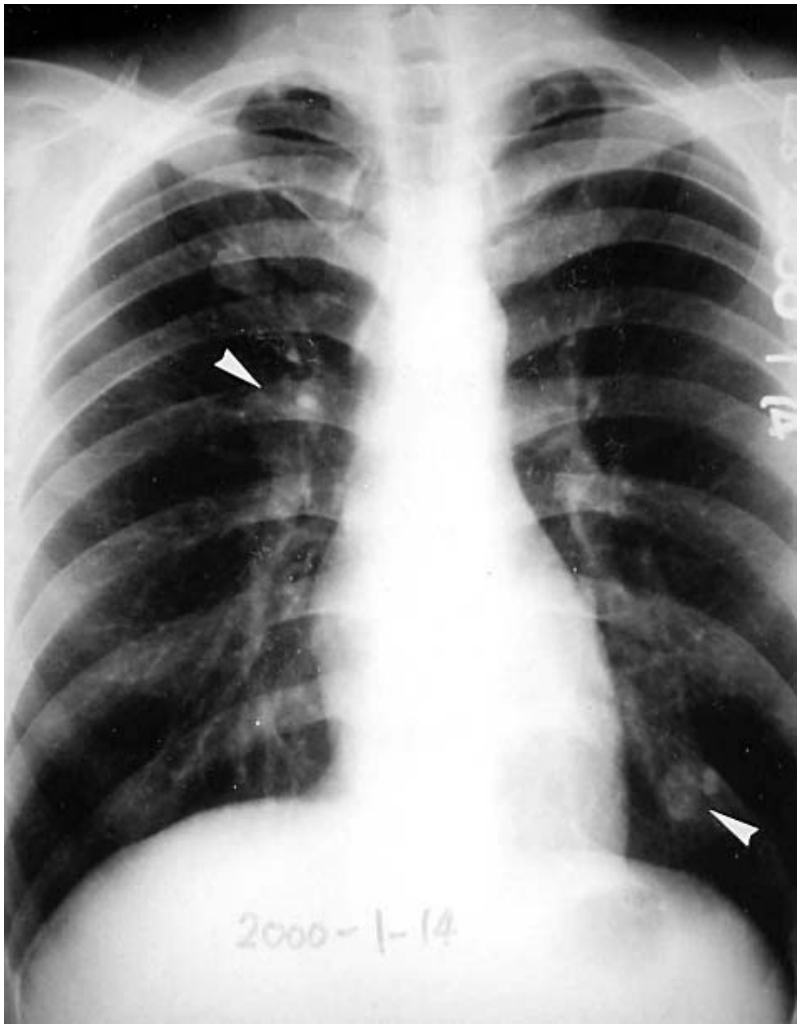

Figure 1 Posterior-anterior chest radiography showing right upper lobe and left lower lobe nodules (arrowheads).

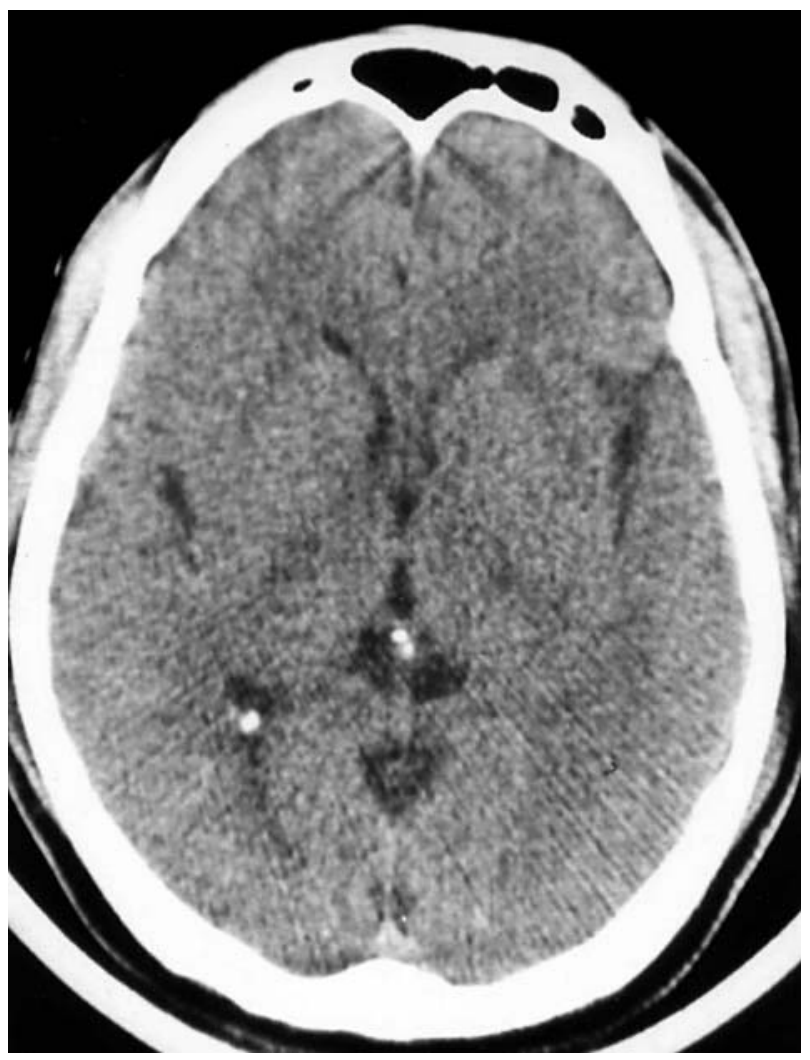

Figure 2 Brain CT revealing diffuse swelling of the bilateral hemispheres. and many of his uncles. Osler-Weber-Rendu disease was diagnosed. In order to avoid any further catastrophic cerebral air embolism or other complications, the patient received a pulmonary angiography and coil embolisation of these PAVMs (fig 4A and B). There were no complication either during or after the procedures. The patient was discharged 26 days later without any sequelae.

\section{DISCUSSION}

The common clinical manifestations of OWRD are epistaxis, dyspnoea, telangiectases, bruit, clubbing, cyanosis, and haemoptysis. ${ }^{1}$ Although the clinical triad of cyanosis, clubbing, and polycythaemia may prompt a search for right-toleft shunt resulting from lesions, the triad may be absent in patients with large PAVM. The condition is most often recognised by chest radiography. The classic roentgenographic appearance of a PAVM is a round or oval mass of uniform density, frequently lobulated (grape shape) but sharply defined, more commonly in the lower lobes, and ranging from 1 to $5 \mathrm{~cm}$ in diameter. Individual PAVM will often reveal feeding vessels on chest radiography, with the arteries radiating from the hilus and the vein deviating toward the left atrium. ${ }^{2}$ Pulmonary angiography is still the diagnostic gold standard for PAVM, but, although associated with minimal risk, it is still an invasive procedure. Noninvasive procedures for evaluating patients with suspected PAVM include measurement of the arterial blood gas tension in room air and with $100 \%$ oxygen for shunt fraction measurement, ${ }^{3}$ detection of orthodeoxia, dynamic CT scanning, contrast echocardiography, radionuclide perfusion lung scanning, and contrast enhanced magnetic resonance angiography. ${ }^{4}$ In this patient, we did not perform the $100 \%$ oxygen method or differential radionuclide scanning for shunt

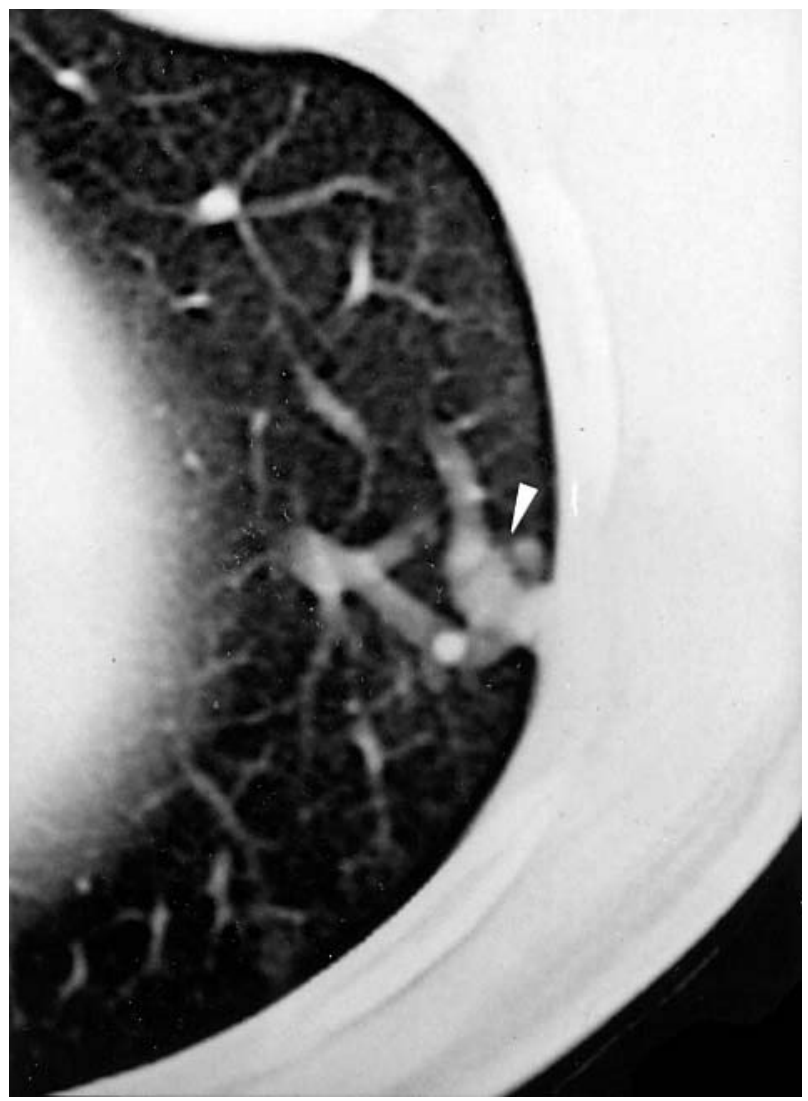

Figure $3 \mathrm{CT}$ of the chest (lung window) revealed left lower lung subpleural arteriovenous communication (arrowhead). 

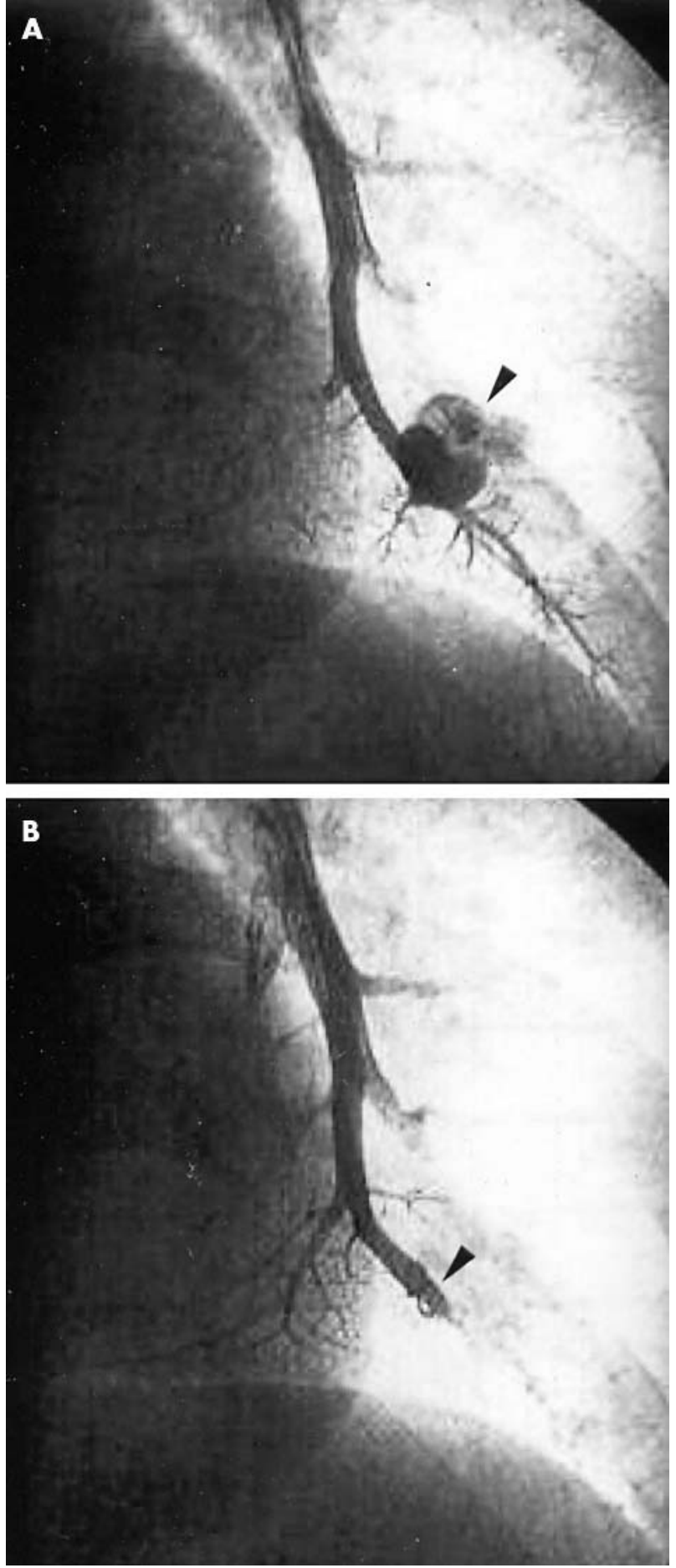

Figure 4 Pulmonary artery angiography showing the tortuous and coiled vascular channels of PAVM (arrowhead) (A), which almost disappeared immediately after embolotherapy (arrowhead) (B).

fraction measurement. But, because of the lack of dyspnoea, orthodeoxsia, cyanosis, and polycythaemia and normal room air arterial blood gas analysis and because of small lesions noted by chest radiography and computed tomography, we hypothesised that the shunt fraction was less than $15 \%$. Colour Doppler ultrasound, which can simultaneously display blood flow information and Doppler spectral wave analysis, is a useful non-invasive procedure for the diagnosis of chest disease. ${ }^{5}$ In this patient, we evaluated the pulmonary lesions with colour Doppler ultrasound and amplitude ultrasound angiography, which showed findings typical of PAVM. ${ }^{6}$

Various complications can occur in patients with PAVM. The most frequently reported relate to the central nervous system and are seen in about $30 \%$ of patients. White and colleagues $^{7}$ evaluated 76 consecutive patients prior to embolotherapy and reported migraine headaches in $43 \%$ of the patients, transient ischaemic attacks in 37\%, cerebrovascular accidents in $18 \%$, brain abscesses in $9 \%$, and seizures in $8 \%$. Although the majority of serious central nervous system events have occurred in symptomatic or cyanotic patients, there have been several reports of previously asymptomatic patients presenting strokes or brain abscesses. ${ }^{89}$ Furthermore, these complications occur almost exclusively in patients with feeding arteries $>3 \mathrm{~mm}$ in diameter. ${ }^{9}$ Paradoxical embolism across a PAVM is the most likely mechanism for major non-infectious cerebrovascular accidents, which are rarely caused by in situ thrombosis related to polycythaemia or by coexisting cerebral arteriovenous malformations. Embolism of infected material is also presumed to account for solitary as well as recurrent brain abscesses. ${ }^{8}$

As diving has become increasingly popular, diving related emergencies have increased dramatically in recent years. The vast majority of dangerous diving related medical conditions, however, are caused by the effects of changes in pressure underwater and the need for breathable air, usually supplied by scuba equipment. The most well known and life threatening complications associated with diving include pulmonary barotraumas, with or without arterial gas embolism (desbaric air embolism), and decompression sickness. ${ }^{10}$ The risk factors for the above complications include rapid decompression in uncontrolled ascents from scuba diving at depths of 3 feet or more, ${ }^{11}$ pre-existing lung pathology such as lung cysts or blebs, ${ }^{12}$ and an intracardiac right-to-left shunt (such as a patent foramen ovale). ${ }^{13}$ Decompression sickness is caused by bubbles of inert gas in the blood and body tissues. Decompression sickness covers a continuum of symptoms and signs ranging from skin itching, achy limb pain, and vague constitutional symptoms to shock and cardiopulmonary arrest. The variety and severity of symptoms depend on bubble volume and location. Rapid decompression with pulmonary overexpansion, and rupture of the alveolar capillary wall, can provide conditions for the development of arterial gas emboli. Another possible mechanism is arterial gas embolism arising in a situation where only venous embolus is referred to as a paradoxical arterial embolism. In most cases of paradoxical embolism related to diving, the right-to-left shunt involved will be a cardiac shunt. A patent foramen ovale (as a right-to-left shunt depending on situational atrial pressure) is present in a quarter of the general population. In contrast, the prevalence

\section{Take home message}

OWRD with PAVM can cause extracardiac right-to-left shunt. We reported a 31 year old male who experienced serious neurological complications during a dive in shallow water. Pulmonary right-to-left shunt and paradoxical cerebral embolism were thought to be responsible for the decompression event. Although we cannot conclude that PAVM is a risk factor for diving related desbaric air embolism, pulmonary angiography with embolotherapy should be performed routinely in this group of patients. Our recommendation is that in these circumstances a diver should refrain from further dives. 


\section{Information Box}

What is already known on this topic

In most cases of paradoxical embolism related to diving, the right-to-left shunt involved will be a cardiac shunt such as a patent foramen ovale. In this 31 year old male, OWRD with PAVM (the cause of extracardiac right-to-left shunt) and paradoxical cerebral embolism were thought to be responsible for the decompression event.

What this study adds

Although we cannot conclude that PAVM is a risk factor for diving related desbaric air embolism, pulmonary angiography with embolotherapy should be performed routinely in this group of patients. Our recommendation is that in these circumstances a diver should refrain from further dives.

of OWRD with concomitant extracardiac shunts varies from 1:5000 to $1: 50000$, making it a rare clinical entity. In this patient, intracardiac shunt was excluded by echocardiography and PAVM was the most probable cause of extracardiac right-to-left shunting.

Finally, the diagnosis of Osler-Weber-Rendu disease (OWRD) with pulmonary arteriovenous malformation was made in a 31 year old man. Although chest radiography and arterial oxygen determination were subtle but typical, computed tomographic, sonographic, and pulmonary angiographic findings confirmed the diagnosis. He experienced serious neurological complications during a dive in shallow water. Pulmonary right-to-left shunt and paradoxical cerebral embolism were thought to be responsible for the decompression event. Although we cannot conclude that PAVM is a risk factor for diving related desbaric air embolism, pulmonary angiography with embolotherapy should be performed routinely in this group of patients. Our recommendation is that in these circumstances a diver should refrain from further dives.

\section{Authors' affiliations}

Y-L Hsu, Far-Eastern Memorial Hospital, Taipei, Taiwan

H-C Wang, P-C Yang, National Taiwan University Hospital, Taipei,

Taiwan

Conflict of interest: none declared.

Correspondence to: Yeong-Long Hsu, Far Eastern Memorial Hospital, Pulmonary and Critical Care Medicine, 2F, No. 25, Lane 461, Chi-Lin Road, Taipei 104, Taiwan; Hsu619@sinamail.com

Received 14 August 2003

Accepted for publication 18 August 2003

\section{REFERENCES}

1 Gossage JR, Kani G. Pulmonary arteriovenous malformations: a state of the art review. Am J Respir Crit Care Med 1998;158:643-61.

2 Dines DE, Seward JB, Bernatz PE. Pulmonary arteriovenous fistula. Mayo Clin Proc 1983;58:176-81.

3 Chilvers ER, Whyte MKB, Jackson JE. Effect of percutaneous transcatheter embolization on pulmonary function, right-to-left shunt and arterial oxygenation in patients with pulmonary arteriovenous malformations. Am Rev Respir Dis 1990;142:420-5.

4 Khalil A, Farres MT, Mangiapan G, et al. Pulmonary arteriovenous malformations. Diagnosis by contrast-enhanced magnetic resonance angiography. Chest 2000;117:1399-403.

5 Yuan A, Chang DB, Yu CJ. Color Doppler sonography of benign and malignant pulmonary masses. Am J Radiol 1994;163:545-9.

6 Wang HC, Kuo PH, Liaw YS, et al. Diagnosis of pulmonary arteriovenous malformations by colour Doppler ultrasound and amplitude ultrasound angiography. Thorax 1998;53:372-6.

7 White RI, Lunch-Nyhan A Jr, Terry P, et al. Pulmonary arteriovenous malformations: techniques and long-term outcome of embolotherapy. Radiology 1988; 169:663-9.

8 Thompson RL, Cattaneo SM, Barnes J. Recurrent brain abscess: manifestation of pulmonary arteriovenous fistula and hereditary hemorrhagic telangiectasis. Chest 1977;72:654-5

9 Hewes RC, Auster M, White RI. Cerebral embolism - first manifestation of pulmonary arteriovenous malformation in patients with hereditary hemorrhagic telangiectasia. Cardiovasc Intervent Radiol 1985;8:151-5.

10 Melamed YM, Shupak A, Bitterman H. Medical problems associated with underwater diving. N Engl J Med 1992:326:30-5.

11 Hardy KR. Diving-related emergencies. Emerg Med Clin North Am 1997; 15:223-40.

12 Reuter M, Tetzlaff K, Warninghoff V, et al. Computed tomography of the chest in diving-related pulmonary barotraumas. $\mathrm{Br} J$ Radiol 1997;70:440-5.

13 Germonpre P, Dendale P, Unger P, et al. Patent foramen ovale and decompression sickness in sports divers. J Appl Physiol 1998;84(5):1622-6. 